\title{
A NOTE ON 2-DIMENSIONAL ARITHMETIC SYMBOLS $\mathrm{ON} \operatorname{Gl}\left(n, \Sigma_{S}\right)$
}

\author{
Fernando Pablos Romo
}

Abstract

The aim of this note is to offer a summary of the definitions and properties of arithmetic symbols on the linear group $\operatorname{Gl}(n, \mathcal{F})$ $-\mathcal{F}$ being an arbitrary discrete valuation field-, and to show that the natural generalizations of the Parshin symbol on an algebraic surface $S$ to the linear group $\operatorname{Gl}\left(n, \Sigma_{S}\right)$ do not allow us to define new 2-dimensional symbols on $S$.

\section{Contents}

1. Introduction 193

2. Preliminaries 195

3. Arithmetic symbols on the linear group $\operatorname{Gl}(n, \mathcal{F}) \quad 196$

4. Parshin symbol on $\operatorname{Gl}\left(n, \Sigma_{S}\right) \quad 203$

$\begin{array}{ll}\text { References } & 207\end{array}$

\section{Introduction}

In 1968, J. Tate [11] introduced the notion of commensurability on vector spaces and gave a definition of the residues of differentials on curves in terms of traces of certain linear operators on infinite-dimensional vector spaces. Furthermore, he proved the residue theorem (the additive reciprocity law) from the finiteness of the cohomology groups $H^{0}\left(C, \mathcal{O}_{C}\right)$ and $H^{1}\left(C, \mathcal{O}_{C}\right)$ when $C$ is a complete curve.

In 1989, a definition of the tame symbol of an algebraic curve from the commutator of a certain central extension of groups was given by

2000 Mathematics Subject Classification. 19F15, 14H05, 11R58.

Key words. Parshin symbol, linear group, algebraic surface, discrete valuation field. This work is partially supported by the DGESYC research contract no. MTM200607618 and Castilla y León regional government contract SA071/04. 
E. Arbarello, C. De Concini and V. G. Kac [1] when the ground field is algebraically closed, and this has been given by the author [9] when it is perfect. In both cases, the reciprocity law of this symbol for complete curves (the "Weil Reciprocity Law") can be also deduced from the finiteness of the cohomology groups $H^{0}\left(C, \mathcal{O}_{C}\right)$ and $H^{1}\left(C, \mathcal{O}_{C}\right)$.

During the last few years, several arithmetic symbols have been studied from commutators of central extensions of groups in different ways, e.g.: in 2002, A. Beilinson, S. Bloch and H. Esnault [2] proved a reciprocity law for the Contou-Carrère symbol [4] from the commutator of a central super-extension of groups defined by using the Gauss-Manin determinant.

The goal of this work is to study, using iterated commutators of central extensions of groups, the possibility of generalizing the Parshin symbol on an algebraic surface [10] to the linear group $\operatorname{Gl}\left(n, \Sigma_{S}\right)$, where $\Sigma_{S}$ is the function field of the surface $S$. In this context, we shall offer a summary of the definitions and properties of arithmetic symbols on the linear group $\operatorname{Gl}(n, \mathcal{F})-\mathcal{F}$ being an arbitrary discrete valuation field-, and we shall show that the natural generalizations of the Parshin symbol on an algebraic surface $S$ to the linear group $\operatorname{Gl}\left(n, \Sigma_{S}\right)$ do not allow us to define new 2-dimensional symbols on $S$.

The organization of the paper is as follows:

Section 2 is devoted to familiarizing readers with the notion of the Parshin symbol on an algebraic surface. Accordingly, its definition and its characterizations as iterated commutators of central extension of groups are given, and the reciprocity laws that this symbol satisfies are written.

Section 3 contains a summary of the definitions and properties of arithmetic symbols on the linear group. Thus, the definition of commensurability on valuation rings, the central extension $\widetilde{G_{\mathcal{V}_{+}}^{\mathcal{V}}}$, the commutator pairing $\{,\}_{\mathcal{V}} \mathcal{V}_{+}$, the arithmetic symbols on $\operatorname{Gl}(n, \mathcal{F})-\mathcal{F}$ being an arbitrary discrete valuation field-, and the reciprocity laws on $\operatorname{Gl}\left(n, \Sigma_{C}\right)$ - where $\operatorname{Gl}\left(n, \Sigma_{C}\right)$ is the function field of a complete, irreducible and non-singular algebraic curve- are briefly introduced.

Finally, Section 4 deals with the main aim of this note: to study the natural generalizations of the Parshin symbol on an algebraic surface to the linear group $\operatorname{Gl}\left(n, \Sigma_{S}\right)$, and to show that we do not obtain new arithmetic symbols from them.

We remark that the proofs of the results offered in Section 3 can be found in $[6]$ and $[8]$. 


\section{Preliminaries}

2.1. The Parshin symbol on an algebraic surface. In 1985, A. N. Parshin [10] introduced a symbol associated with a sequence $x \in C \subset S$, where $C$ is a curve on an algebraic surface $S$ over an algebraically closed field $k$, and $x$ is a closed point of $C$. If $f, g$ and $h$ are three invertible functions on $S$, the expression of the symbol is:

$$
\langle f, g, h\rangle_{(x, C)}=(-1)^{\alpha_{(x, C)}} \cdot\{f, g, h\}_{(x, C)} \in k^{*},
$$

where

$$
\{f, g, h\}_{(x, C)}=\left(\frac{f^{v_{C}(g) \cdot v_{x}^{z}(h)-v_{C}(h) \cdot v_{x}^{z}(g)}}{g^{v_{C}(f) \cdot v_{x}^{z}(h)-v_{C}(h) \cdot v_{x}^{z}(f)}} \cdot h^{v_{C}(f) \cdot v_{x}^{z}(g)-v_{C}(g) \cdot v_{x}^{z}(f)}\right)_{\left.\right|_{C}}(p),
$$

and

$$
\begin{aligned}
\alpha_{(x, C)}= & v_{C}(f) \cdot v_{C}(g) \cdot v_{x}^{z}(h)+v_{C}(f) \cdot v_{C}(h) \cdot v_{x}^{z}(g) \\
& +v_{C}(g) \cdot v_{C}(h) \cdot v_{x}^{z}(f)+v_{C}(f) \cdot v_{x}^{z}(g) \cdot v_{x}^{z}(h) \\
& +v_{C}(g) \cdot v_{x}^{z}(f) \cdot v_{x}^{z}(h)+v_{C}(h) \cdot v_{x}^{z}(f) \cdot v_{x}^{z}(g),
\end{aligned}
$$

$v_{C}$ being the discrete valuation induced by $C$ (a codimension-one subvariety of $S)$, and $v_{x}^{z}(\varphi)$ is the valuation $v_{2}(\varphi)$ introduced in $[\mathbf{3}]$, which is the order of $\left(\varphi \cdot z^{-v_{C}(\varphi)}\right)_{\left.\right|_{C}}$ at $x$ in $C$, for a function $z$ on $S$ such that $v_{C}(z)=1$.

It should be noted that this explicit expression is not completely due to A. N. Parshin: i.e., the higher dimensional tame symbol was defined by Parshin up to the sign, and the full definition, including the sign, was given by I. B. Fesenko in his thesis in 1986 and was published in 1988 [5] (for the English translation, see $[\mathbf{1 2}]$ ).

This symbol is independent of the choice of $z$, and it satisfies the reciprocity laws:

$$
\begin{aligned}
& \prod_{x}\langle f, g, h\rangle_{(x, C)}=1, \\
& \prod_{C}\langle f, g, h\rangle_{(x, C)}=1,
\end{aligned}
$$

where $C$ is a complete, irreducible and non-singular curve in (2.2), and the second reciprocity law is the product over all irreducible curves containing a fixed point $x \in S$.

If $\Sigma_{S}$ is the function field of $S$, it is a discrete valuation field with the valuation $v_{C}$, whose residue class field is $\Sigma_{C}$ (the function field of $C$ ). 
Thus, it follows from the characterization of the tame symbol [7] that there exists a central extension of groups:

$$
1 \longrightarrow \Sigma_{C}^{*} \longrightarrow \widetilde{\Sigma_{S}^{*}} \longrightarrow \Sigma_{S}^{*} \longrightarrow 1,
$$

such that the value of its commutator is:

$$
\{f, g\}_{C}^{S}=\left(\frac{f^{v_{C}(g)}}{g^{v_{C}(f)}}\right)_{\left.\right|_{C} \in \Sigma_{C}^{*}}
$$

for each $f, g \in \Sigma_{S}^{*}$.

If $\{,\}_{A_{x}}^{K_{x}}$ is the commutator of the central extension studied in [1] and [9] that coincides, up to the sign, with the tame symbol associated with the closed point $x \in C ; z$ is again a function $z$ on $S$ such that $v_{C}(z)=1$, and $t$ is a function on $C$ such that $v_{x}(t)=1$, then we can interpret the map $\langle,,\rangle_{(x, C)}$ as iterated commutators in a double way:

- If $\{f, g, h\}_{x, C}^{z}=\left[\left\{\{f, z\}_{C}^{S},\{g, z\}_{C}^{S}\right\}_{A_{x}}^{K_{x}}\right]^{-v_{C}(h)}$, one has that:

$$
\{f, g, h\}_{(x, C)}=\{f, g, h\}_{x, C}^{z} \cdot\{g, h, f\}_{x, C}^{z} \cdot\{h, f, g\}_{x, C}^{z} .
$$

- If $\{f, g, h\}_{x, C}^{z, t}=\left[\left\{\{f, g\}_{C}^{S}, t\right\}_{A_{x}}^{K_{x}}\right]_{x}^{v_{x}^{z}(h)}$, one has also that:

$$
\{f, g, h\}_{(x, C)}=\{f, g, h\}_{x, C}^{z, t} \cdot\{g, h, f\}_{x, C}^{z, t} \cdot\{h, f, g\}_{x, C}^{z, t} .
$$

\section{Arithmetic symbols on the linear group $\operatorname{Gl}(n, \mathcal{F})$}

3.1. Commensurability on valuation rings. In this subsection, domain refers to an integral commutative with unit element ring. Let $\mathcal{F}$ be a discrete valuation field and let $\mathcal{K}(v)$ be its residue class field. The valuation ring associated with $v$ is again denoted by $\mathcal{O}_{v}$, and $\mathfrak{m}_{v}$ is its maximal ideal.

Let $\mathcal{V}$ be an $\mathcal{O}_{v}$-module and let $\mathcal{M}, \mathcal{N}$ be two $\mathcal{O}_{v}$-submodules of $\mathcal{V}$. Note that $\mathcal{O}_{v}$ is a local principal ideal domain.

Definition 3.1. $\mathcal{M}$ and $\mathcal{N}$ are said to be commensurable if $\mathcal{M}+\mathcal{N} / \mathcal{M} \cap \mathcal{N}$ is a torsion finitely generated $\mathcal{O}_{v}$-module. We shall use the symbol $\mathcal{M} \sim$ $\mathcal{N}$ to denote commensurable submodules.

Similar to $[\mathbf{1}]$, one has that:

(1) If $\mathcal{M} \sim \mathcal{N}$ and $\mathcal{N} \sim \mathcal{P}$, then $\mathcal{M}+\mathcal{N}+\mathcal{P} / \mathcal{M} \cap \mathcal{N} \cap \mathcal{P}$ is a torsion finitely generated $\mathcal{O}_{v}$-module.

(2) Commensurability is an equivalence relation.

(3) Let $\mathcal{M}, \mathcal{N}, \mathcal{M}^{\prime}, \mathcal{N}^{\prime}$ be submodules of $\mathcal{V}$ and let us assume that $\mathcal{M} \sim$ $\mathcal{M}^{\prime}$ and $\mathcal{N} \sim \mathcal{N}^{\prime}$. Then, $\mathcal{M}+\mathcal{N} \sim \mathcal{M}^{\prime}+\mathcal{N}^{\prime}$ and $\mathcal{M} \cap \mathcal{N} \sim \mathcal{M}^{\prime} \cap \mathcal{N}^{\prime}$. 
Moreover, if $\mathcal{W}$ is a $\mathcal{O}_{v}$-module, we use $\mathcal{G}_{\mathfrak{m}_{v}}(\mathcal{W})$ to denote its graded $\mathcal{K}(v)$-module induced by the $\mathfrak{m}_{v}$-filtration $\left\{\mathfrak{m}_{v}^{n} \mathcal{W}\right\}_{n \geq 0}$. Given commensurable submodules $\mathcal{M}$ and $\mathcal{N}$ of $\mathcal{V}$, we set

$$
[\mathcal{M} \mid \mathcal{N}]_{\mathfrak{m}_{v}}=\operatorname{dim}_{\mathcal{K}(v)} \mathcal{G}_{\mathfrak{m}_{v}}(\mathcal{M} / \mathcal{M} \cap \mathcal{N})-\operatorname{dim}_{\mathcal{K}(v)} \mathcal{G}_{\mathfrak{m}_{v}}(\mathcal{N} / \mathcal{M} \cap \mathcal{N})
$$

Remark 3.2. Let $\mathcal{M}, \mathcal{N}$ and $\mathcal{P}$ be pairwise commensurable submodules of $\mathcal{V}$. Thus,

$$
[\mathcal{M} \mid \mathcal{N}]_{\mathfrak{m}_{v}}+[\mathcal{N} \mid \mathcal{P}]_{\mathfrak{m}_{v}}=[\mathcal{M} \mid \mathcal{P}]_{\mathfrak{m}_{v}}
$$

3.2. The central extension $\widetilde{G_{\mathcal{V}_{+}}^{\mathcal{V}}}$. Let $\mathcal{V}$ again be an $\mathcal{O}_{v}$-module and let $\mathcal{V}_{+}$be a fixed $\mathcal{O}_{v}$-submodule of $\mathcal{V}$.

Definition 3.3. Define the group $G_{\mathcal{V}_{+}}^{\mathcal{V}} \subseteq \operatorname{Aut}_{\mathcal{O}_{v}}(\mathcal{V})$ by

$G_{\mathcal{V}_{+}}^{\mathcal{V}}=\left\{\tau \in \operatorname{Aut}_{\mathcal{O}_{v}}(\mathcal{V})\right.$ such that $\tau\left(\mathcal{V}_{+}\right) \sim \mathcal{V}_{+}$and $\left.\tau^{-1}\left(\mathcal{V}_{+}\right) \sim \mathcal{V}_{+}\right\}$

To keep the notations of [9], if $\tau \in G_{\mathcal{V}_{+}}^{\mathcal{V}}$ and $\Lambda$ is the maximal exterior power, we set

$$
\operatorname{Det} \mathcal{C}_{\tau \mathcal{V}_{+}}^{\bullet}=\Lambda \mathcal{G}_{\mathfrak{m}_{v}}\left(\mathcal{V}_{+} / \mathcal{V}_{+} \cap \tau \mathcal{V}_{+}\right) \underset{\mathcal{K}(v)}{\otimes} \Lambda\left[\mathcal{G}_{\mathfrak{m}_{v}}\left(\tau \mathcal{V}_{+} / \mathcal{V}_{+} \cap \tau \mathcal{V}_{+}\right)\right]^{*}
$$

which is a $\mathcal{K}(v)$-vector space of dimension one.

If $\tau \mathcal{V}_{+}=\mathcal{V}_{+}$, then $\operatorname{Det} \mathcal{C}_{\tau}^{\bullet} \mathcal{V}_{+}=\mathcal{K}(v)^{*}$

Moreover, from the computations made in $[\mathbf{1}],[\mathbf{7}]$ and $[\mathbf{9}]$ one deduces the existence of a map

$$
\operatorname{Det} \mathcal{C}_{\tau \mathcal{V}_{+}}^{\bullet} \underset{\mathcal{K}(v)}{\otimes} \operatorname{Det} \mathcal{C}_{\sigma \mathcal{V}_{+}}^{\bullet} \stackrel{\varphi_{\tau}^{\sigma}}{\longrightarrow} \operatorname{Det} \mathcal{C}_{\tau \sigma \mathcal{V}_{+}}^{\bullet}
$$

that we shall write as $\varphi_{\tau}^{\sigma}\left(s_{1} \otimes s_{2}\right)=s_{1} \cdot \bar{\tau}\left(s_{2}\right)$, where $\bar{\tau}\left(s_{2}\right)$ is an element of

$$
\Lambda \mathcal{G}_{\mathfrak{m}_{v}}\left(\tau \mathcal{V}_{+} / \tau \mathcal{V}_{+} \cap \tau \sigma \mathcal{V}_{+}\right) \underset{\mathcal{K}(v)}{\otimes} \Lambda\left[\mathcal{G}_{\mathfrak{m}_{v}}\left(\tau \sigma \mathcal{V}_{+} / \tau \mathcal{V}_{+} \cap \tau \sigma \mathcal{V}_{+}\right)\right]^{*}
$$

Let us now consider the set

$$
\widetilde{G_{\mathcal{V}_{+}}^{\mathcal{V}}}=\left\{(\tau, s) \text { with } \tau \in G_{\mathcal{V}_{+}}^{\mathcal{V}}, 0 \neq s \in \operatorname{Det} \mathcal{C}_{\tau \mathcal{V}_{+}}^{\bullet}\right\}
$$

together with the operation

$$
\left(\tau, s_{1}\right) \cdot\left(\sigma, s_{2}\right)=\left(\tau \sigma, s_{1} \cdot \bar{\tau}\left(s_{2}\right)\right) .
$$

It follows from the statements of $[\mathbf{1}],[\mathbf{7}]$ and $[\mathbf{9}]$ that $\widetilde{G_{\mathcal{V}}}$, with this operation, forms a group and there exists a central extension of groups:

$$
1 \longrightarrow \mathcal{K}(v)^{*} \stackrel{i}{\longrightarrow} \widetilde{G_{\mathcal{V}_{+}}^{\mathcal{V}}} \stackrel{\pi}{\longrightarrow} G_{\mathcal{V}_{+}}^{\mathcal{V}} \longrightarrow 1
$$

where $i(\lambda)=(\mathrm{Id}, \lambda)$ and $\pi(\tau, s)=\tau$. 
Definition 3.4. Given an element $\tau \in G_{\mathcal{V}_{+}}^{\mathcal{V}}$, we shall call the integer number

$$
i_{\mathcal{K}(v)}\left(\tau, \mathcal{V}_{+}\right)=\left[\mathcal{V}_{+} \mid \tau \mathcal{V}_{+}\right]_{\mathfrak{m}_{v}}
$$

the "index of $\tau$ over $\mathcal{V}_{+}$".

Remark 3.5. Note that:

$i_{\mathcal{K}(v)}\left(\tau, \mathcal{V}_{+}\right)=\operatorname{dim}_{\mathcal{K}(v)} \mathcal{G}_{\mathfrak{m}_{v}}\left(\mathcal{V}_{+} / \tau \mathcal{V}_{+} \cap \mathcal{V}_{+}\right)-\operatorname{dim}_{\mathcal{K}(v)} \mathcal{G}_{\mathfrak{m}_{v}}\left(\mathcal{V}_{+} / \tau^{-1} \mathcal{V}_{+} \cap \mathcal{V}_{+}\right)$

Moreover, if $\mathcal{M} \sim \mathcal{V}_{+}$, then $i_{\mathcal{K}(v)}(\tau, \mathcal{M})=i_{\mathcal{K}(v)}\left(\tau, \mathcal{V}_{+}\right)$.

3.3. The commutator pairing $\{,\}_{\mathcal{V}_{+}}$. If $\tau$ and $\sigma$ are two commuting elements of $G_{\mathcal{V}_{+}}^{\mathcal{V}}$ and $\widetilde{\tau}, \widetilde{\sigma} \in \widetilde{G_{\mathcal{V}}^{\mathcal{V}}}$ are elements such that $\pi(\widetilde{\tau})=\tau$ and $\pi(\widetilde{\sigma})=\sigma$, then one has a commutator pairing:

$$
\{\tau, \sigma\}_{\mathcal{V}_{+}}^{\mathcal{V}}=\widetilde{\tau} \cdot \widetilde{\sigma} \cdot \widetilde{\tau}^{-1} \cdot \widetilde{\sigma}^{-1} \in \mathcal{K}(v)^{*} .
$$

Fix elements $\sigma, \sigma^{\prime}, \tau, \tau^{\prime} \in G_{\mathcal{V}_{+}}^{\mathcal{V}}$ such that the $\sigma^{\prime}$ 's commute with the $\tau$ 's. (But we need assume neither that $\sigma \sigma^{\prime}=\sigma^{\prime} \sigma$ nor that $\tau \tau^{\prime}=\tau^{\prime} \tau$.) Hence, from the definition of the commutator pairing, from the construction of the central extension $\widetilde{G_{\mathcal{V}}^{\mathcal{V}}}$ or from the statements of [8], the following relations hold:

- $\{\sigma, \sigma\} \mathcal{V}_{+}=1$

- $\{\sigma, \tau\} \mathcal{V}_{\mathcal{V}_{+}}^{\mathcal{V}}=\left(\{\tau, \sigma\} \mathcal{V}_{\mathcal{V}_{+}}\right)^{-1}$.

- $\left\{\sigma \sigma^{\prime}, \tau\right\} \mathcal{V}_{+}=\{\sigma, \tau\} \mathcal{V}_{+}\left\{\sigma^{\prime}, \tau\right\} \mathcal{V}_{+}$.

- $\left\{\sigma, \tau \tau^{\prime}\right\} \mathcal{V}_{+}=\{\sigma, \tau\} \mathcal{V}_{+}\left\{\sigma, \tau^{\prime}\right\} \mathcal{V}_{+}$.

- $\sigma \mathcal{V}_{+}=\mathcal{V}_{+}=\tau \mathcal{V}_{+} \Rightarrow\{\sigma, \tau\}^{\mathcal{V}} \mathcal{V}_{+}=1$

- If $\mathcal{V}_{+}=\{0\}$ or $\mathcal{V}_{+}=\mathcal{V}$, then $\{\sigma, \tau\} \mathcal{V}_{+}=1$

- $\{\sigma, \tau\} \mathcal{V}_{\mathcal{V}_{+}}$depends only on the commensurability class of $\mathcal{V}_{+}$.

- Suppose $\mathcal{V}$ is equipped with a direct sum decomposition $\mathcal{V}=\mathcal{V}_{0} \oplus$ $\mathcal{V}_{1}$. Put $\mathcal{V}_{i+}:=\mathcal{V}_{i} \cap \mathcal{V}_{+}$for $i=0,1$ and assume that $\mathcal{V}_{+}=\mathcal{V}_{0+} \oplus$ $\mathcal{V}_{1+}$. Let commuting elements $\sigma_{0}, \sigma_{1} \in G_{\mathcal{V}_{+}}^{\mathcal{V}}$ be given such that

$$
\sigma_{i}\left|\mathcal{V}_{0} \in G_{\mathcal{V}_{0+}}^{\mathcal{V}_{0}}, \quad \sigma_{i}\right| \mathcal{V}_{1}=1
$$

for $i=0,1$. Then we have

$$
\left\{\sigma_{0}\left|\mathcal{V}_{0}, \sigma_{1}\right| \mathcal{V}_{0}\right\}_{\mathcal{V}_{0+}}^{\mathcal{V}_{0}}=\left\{\sigma_{0}, \sigma_{1}\right\}_{\mathcal{V}_{+}}^{\mathcal{V}}
$$


- Again suppose $\mathcal{V}$ is equipped with a direct sum decomposition $\mathcal{V}=$ $\mathcal{V}_{0} \oplus \mathcal{V}_{1}$, put $\mathcal{V}_{i+}:=\mathcal{V}_{i} \cap \mathcal{V}_{+}$for $i=0,1$ and assume that $\mathcal{V}_{+}=$ $\mathcal{V}_{0+} \oplus \mathcal{V}_{1+}$. Let $\sigma_{0}, \sigma_{1} \in G_{\mathcal{V}_{+}}$be given such that

$$
\left.\sigma_{i}\right|_{\mathcal{V}_{i}} \in G_{\mathcal{V}_{i+}}^{\mathcal{V}_{i}},\left.\quad \sigma_{i}\right|_{\mathcal{V}_{1-i}}=1
$$

for $i=0,1$. (Necessarily $\sigma_{0}$ and $\sigma_{1}$ commute.) Then we have

$$
\left\{\sigma_{0}, \sigma_{1}\right\}_{\mathcal{V}_{+}}^{\mathcal{V}}=(-1)^{\alpha_{0} \alpha_{1}}
$$

where

$$
\alpha_{i}:=i_{\mathcal{K}(v)}\left(\left.\sigma_{i}\right|_{\mathcal{V}_{i}}, \mathcal{V}_{i+}\right)=i_{\mathcal{K}(v)}\left(\sigma_{i}, \mathcal{V}_{+}\right)
$$

for $i=0,1$.

- If $\mathcal{V}=\mathcal{V}_{0} \oplus \mathcal{V}_{0}$ and $\mathcal{V}_{+}=\mathcal{V}_{0+} \oplus \mathcal{V}_{1+}$, such that $\mathcal{V}_{0}$ and $\mathcal{V}_{1}$ are invariant by the action of two commuting elements $\tau, \sigma \in G_{\mathcal{V}_{+}}^{\mathcal{V}}$ and $\tau\left|\mathcal{V}_{i}, \sigma\right|_{\mathcal{V}_{i}} \in G_{\mathcal{V}_{i+}}^{\mathcal{V}_{i}}$, then

$$
\{\tau, \sigma\}_{\mathcal{V}_{+}}^{\mathcal{V}}=(-1)^{\alpha} \cdot\left\{\left.\tau\right|_{\mathcal{V}_{0}},\left.\sigma\right|_{\mathcal{V}_{0}}\right\}_{\mathcal{V}_{0+}}^{\mathcal{V}_{0}} \cdot\left\{\left.\tau\right|_{\mathcal{V}_{1}},\left.\sigma\right|_{\mathcal{V}_{1}}\right\}_{\mathcal{V}_{1+}}^{\mathcal{V}_{1}}
$$

with $\alpha=i_{\mathcal{K}(v)}\left(\left.\tau\right|_{\mathcal{V}_{0}}, \mathcal{V}_{0+}\right) \cdot i_{\mathcal{K}(v)}\left(\left.\sigma\right|_{\mathcal{V}_{1}}, \mathcal{V}_{1+}\right)+i_{\mathcal{K}(v)}\left(\left.\tau\right|_{\mathcal{V}_{1}}, \mathcal{V}_{1+}\right)$. $i_{\mathcal{K}(v)}\left(\left.\sigma\right|_{\mathcal{V}_{0}}, \mathcal{V}_{0+}\right)$.

- If $\mathcal{M}$ and $\mathcal{N}$ are two $\mathcal{O}_{v}$-submodules of $\mathcal{V}$, for all commuting elements $\tau, \sigma \in G_{\mathcal{M}}^{\mathcal{V}} \cap G_{\mathcal{N}}^{\mathcal{V}}$, one has that $\tau, \sigma \in G_{\mathcal{M}+\mathcal{N}}^{\mathcal{V}} \cap G_{\mathcal{M} \cap \mathcal{N}}^{\mathcal{V}}$ and:

$$
\{\tau, \sigma\}_{\mathcal{M}}^{\mathcal{V}} \cdot\{\tau, \sigma\}_{\mathcal{N}}^{\mathcal{V}}=(-1)^{\beta} \cdot\{\tau, \sigma\}_{\mathcal{M}+\mathcal{N}}^{\mathcal{V}} \cdot\{\tau, \sigma\}_{\mathcal{M} \cap \mathcal{N}}^{\mathcal{V}}
$$

where

$$
\begin{aligned}
\beta= & i_{\mathcal{K}(v)}(\mathcal{M}, \tau) \cdot i_{\mathcal{K}(v)}(\sigma, \mathcal{N})+i_{\mathcal{K}(v)}(\tau, \mathcal{N}) \cdot i_{\mathcal{K}(v)}(\sigma, \mathcal{M}) \\
& +i_{\mathcal{K}(v)}(\tau, \mathcal{M}+\mathcal{N}) \cdot i_{\mathcal{K}(v)}(\sigma, \mathcal{M} \cap \mathcal{N}) \\
& +i_{\mathcal{K}(v)}(\tau, \mathcal{M} \cap \mathcal{N}) \cdot i_{\mathcal{K}(v)}(\sigma, \mathcal{M}+\mathcal{N})
\end{aligned}
$$

3.4. Arithmetic symbols on $\operatorname{Gl}(n, \mathcal{F})$. With the above notations, for a positive integer number, $n$, let us consider the $\mathcal{O}_{v}$-modules:

$$
\begin{aligned}
& \mathcal{V}^{n}=\left(\widehat{\mathcal{O}_{v}}\right)_{0} \oplus \ldots \stackrel{n}{n} . \oplus\left(\widehat{\mathcal{O}_{v}}\right)_{0} \\
& \mathcal{V}_{+}^{n}=\widehat{\mathcal{O}_{v}} \oplus \ldots \stackrel{n}{\ldots} \ldots \widehat{\mathcal{O}_{v}} \subseteq \mathcal{V}^{n} .
\end{aligned}
$$

If we denote by $\operatorname{Gl}(n, \mathcal{F})$ the linear group of rank $n$ (that is, the group of invertible $n \times n$ matrices with entries in $\mathcal{F}$ ), it is clear that $\operatorname{Gl}(n, \mathcal{F}) \subseteq G_{\mathcal{V}_{+}^{n}}^{\mathcal{V}^{n}}$ 
Thus, from the extension (3.1) one has a central extension of groups:

$$
1 \longrightarrow \mathcal{K}(v)^{*} \stackrel{i}{\longrightarrow} \widehat{\operatorname{Gl}(n, \mathcal{F})} \stackrel{\pi}{\longrightarrow} \operatorname{Gl}(n, \mathcal{F}) \longrightarrow 1,
$$

and if $\tau$ and $\sigma$ are two commuting elements of $\operatorname{Gl}(n, \mathcal{F})$ and $\widetilde{\tau}, \widetilde{\sigma} \in$ $\widetilde{\operatorname{Gl}(n, \mathcal{F})}$ are elements such that $\pi(\widetilde{\tau})=\tau$ and $\pi(\widetilde{\sigma})=\sigma$, then one has a commutator pairing:

$$
\{\tau, \sigma\} \mathcal{V}_{+}^{\mathcal{V}^{n}}=\widetilde{\tau} \cdot \widetilde{\sigma} \cdot \widetilde{\tau}^{-1} \cdot \widetilde{\sigma}^{-1} \in \mathcal{K}(v)^{*}
$$

Example 1. Let us consider the commuting matrices $\tau_{f_{i}}, \tau_{g_{j}} \in \operatorname{Gl}(n, \mathcal{F})$, where:

$$
\tau_{f_{i}}=\left(\begin{array}{ccc}
f_{1} & \ldots & 0 \\
\vdots & \ddots & \vdots \\
0 & \ldots & f_{n}
\end{array}\right) \text { and } \tau_{g_{j}}=\left(\begin{array}{ccc}
g_{1} & \ldots & 0 \\
\vdots & \ddots & \vdots \\
0 & \ldots & g_{n}
\end{array}\right) \text {, }
$$

with $f_{i}, g_{j} \in \mathcal{F}^{*}$.

It follows from the properties of the commutator pairing and the statements of $[7]$ that:

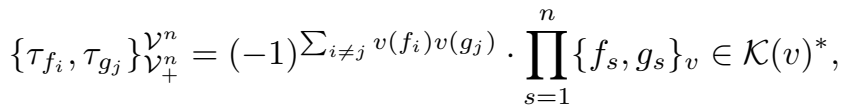

where $\{,\}_{v}: \mathcal{F}^{*} \times \mathcal{F}^{*} \rightarrow k(v)^{*}$ is the 2-cocycle:

$$
\{f, g\}_{v}=\frac{f^{v(g)}}{g^{v(f)}} \quad\left(\bmod \cdot \mathfrak{m}_{v}\right) .
$$

According to the statements of [8], one has that:

Lemma 3.6. If $\tau \in \operatorname{Gl}(n, \mathcal{F})$, then

$$
i_{\mathcal{K}(v)}\left(\tau, \mathcal{V}_{+}^{n}\right)=v(\operatorname{det} \tau)
$$

Moreover, regarding $\mathcal{F}^{*}$ as a subgroup of $\operatorname{Gl}(n, \mathcal{F})$ by means of the diagonal embedding

$$
f \longmapsto \sigma_{f}^{n}:=\left(\begin{array}{ccc}
f & \ldots & 0 \\
\vdots & \ddots & \vdots \\
0 & \ldots & f
\end{array}\right)
$$

one has that $\mathcal{F}^{*}=Z(\operatorname{Gl}(n, \mathcal{F}))$ and, therefore, there exists a commutator map

$$
\{,\} \mathcal{V}_{+}^{\mathcal{V}_{+}^{n}}: \mathcal{F}^{*} \times \operatorname{Gl}(n, \mathcal{F}) \longrightarrow k(v)^{*}
$$

The explicit expression of this commutator map is: 
Proposition 3.7. For all $f \in \mathcal{F}^{*}$ and $\tau \in \operatorname{Gl}(n, \mathcal{F})$, one has that

$$
\begin{aligned}
\left.\left\{\sigma_{f}^{n}, \tau\right\}\right\}_{\mathcal{V}_{+}^{n}}^{\mathcal{V}^{n}} & =(-1)^{(n-1) \cdot v(f) \cdot v(\operatorname{det} \tau)} \cdot\{f, \operatorname{det} \tau\}_{v} \\
& =(-1)^{(n-1) \cdot v(f) \cdot v(\operatorname{det} \tau)} \cdot\left(\frac{f^{v(\operatorname{det} \tau)}}{[\operatorname{det} \tau]^{v(f)}}\left(\bmod \cdot \mathfrak{m}_{v}\right)\right) .
\end{aligned}
$$

Remark 3.8. When $n=1$, the above expression is the commutator $\{,\}_{v}$ that determines the tame symbol on an arbitrary discrete valuation field $\mathcal{F}[\mathbf{7}]$, and, when $n=2$, if $X$ is an algebraic curve over an algebraically closed field $k, x \in X$ is a closed point, $\mathcal{F}=\Sigma_{X}$ (the function field of $X$ ), and $v=v_{x}$ (the discrete valuation induced by the point $x$ ), the statement of Proposition 3.7 coincides with the characterization of the commutator map $\{,\}_{A_{x}^{2}}^{K^{2}}: \Sigma_{X}^{*} \times \operatorname{Gl}\left(2, \Sigma_{X}\right) \rightarrow k^{*}$ obtained in [6].

We can now define a generalization of the tame symbol from the commutator $\{,\}_{\mathcal{V}_{+}^{n}}^{\mathcal{L}^{n}}$.

Definition 3.9. With the previous notations, we shall use the term "tame symbol" on $\operatorname{Gl}(n, \mathcal{F})$ associated with $v$ to refer to the assignment:

$$
(\tau, \varphi)_{v}^{n}=(-1)^{v(\operatorname{det} \tau) \cdot v(\operatorname{det} \varphi)} \cdot\{\tau, \sigma\}_{\mathcal{V}_{+}^{n}}^{\mathcal{V}^{n}} \in \mathcal{K}(v)^{*},
$$

where $\tau, \varphi$ are two commuting matrices of $\operatorname{Gl}(n, \mathcal{F})$.

Example 2. Let us consider a non-singular and irreducible curve $C$ over an algebraically closed field $k$ and a closed point $x \in C$. If $\Sigma_{C}$ is the function field of $C$ and $v_{x}$ is the discrete valuation on $\Sigma_{C}$ associated with $x$, by using the above method we can define the "tame symbol on $\operatorname{Gl}\left(n, \Sigma_{C}\right)$ associated with $x "$, whose explicit expression is:

$$
(\tau, \sigma)_{x}^{n}=(-1)^{v_{x}(\operatorname{det} \tau) \cdot v_{x}(\operatorname{det} \sigma)} \cdot\{\tau, \sigma\}_{A_{x}^{n}}^{K_{x}^{n}} \in k^{*},
$$

where $\tau, \sigma \in \operatorname{Gl}\left(n, \Sigma_{C}\right)$ are commuting matrices, $\widehat{\mathcal{O}_{x}}$ is the completion of the local ring $\mathcal{O}_{x},\left(\widehat{\mathcal{O}_{x}}\right)_{0}$ is the field of fractions of $\widehat{\mathcal{O}_{x}}, A_{x}^{n}=\widehat{\mathcal{O}_{x}} \oplus \ldots \stackrel{n}{\ldots} \oplus \widehat{\mathcal{O}_{x}}$ and $K_{x}^{n}=\left(\widehat{\mathcal{O}_{x}}\right)_{0} \oplus \ldots \stackrel{n}{n} \oplus \oplus\left(\widehat{\mathcal{O}_{x}}\right)_{0}$.

Note that if $n=1$, for all $f, g \in \Sigma_{C}^{*}$ one has that $\{f, g\}_{A_{x}}^{K_{x}}=\frac{f^{v_{x}(g)}}{g^{v_{x}(f)}}(x)$ and, therefore,

$$
(f, g)_{x}=(-1)^{v_{x}(f) \cdot v_{x}(g)} \cdot \frac{f^{v_{x}(g)}}{g^{v_{x}(f)}}(x) \in k^{*}
$$

is the tame symbol associated with the closed point $x \in C$. 
Moreover, let us consider an abelian group $G$ and a morphism of groups $\psi: \mathcal{K}(v)^{*} \rightarrow G$. The central extension (3.2) induces another central extension of groups:

$$
1 \longrightarrow G \stackrel{i}{\longrightarrow} \operatorname{Gl}(n, \mathcal{F})_{\psi} \stackrel{\pi}{\longrightarrow} \operatorname{Gl}(n, \mathcal{F}) \longrightarrow 1,
$$

whose commutator is

$$
\{\tau, \sigma\}_{\mathcal{V}_{+}^{n}, \psi}^{\mathcal{V}^{n}}=\widetilde{\tau} \cdot \widetilde{\sigma} \cdot \widetilde{\tau}^{-1} \cdot \widetilde{\sigma}^{-1},
$$

$\tau, \sigma \in \operatorname{Gl}(n, \mathcal{F})$ being two commuting matrices, $\widetilde{\tau}, \widetilde{\sigma} \in \widetilde{\operatorname{Gl}(n, \mathcal{F})})_{\psi}, \pi(\widetilde{\tau})=\tau$ and $\pi(\widetilde{\sigma})=\sigma$. It is clear that $\{,\}_{\mathcal{V}_{+}^{n}, \psi}^{\mathcal{V}^{n}}=\psi \circ\{,\}_{\mathcal{V}_{+}^{n}}^{\mathcal{V}^{n}}$.

Definition 3.10. With the above notation, given an abelian group $G$ and a morphism of groups $\psi: \mathcal{K}(v)^{*} \rightarrow G$, we shall use the term "tame symbol on $\operatorname{Gl}(n, \mathcal{F})$ associated with $v$ and the morphism $\psi$ " to refer to the assignment:

$$
(\tau, \varphi)_{v, \psi}^{n}=\psi\left[(-1)^{v(\operatorname{det} \tau) \cdot v(\operatorname{det} \varphi)}\right] \cdot\{\tau, \sigma\}_{\mathcal{V}_{+}^{n}, \psi}^{\mathcal{V}^{n}} \in G,
$$

where $\tau, \varphi$ are two commuting matrices of $\operatorname{Gl}(n, \mathcal{F})$.

3.5. Reciprocity laws on $\operatorname{Gl}\left(\boldsymbol{n}, \boldsymbol{\Sigma}_{C}\right)$. Let $C$ be a non-singular and irreducible curve over an algebraically closed field $k$. For each closed point $x \in C$ we consider the $k$-vector spaces $A_{x}=\widehat{\mathcal{O}_{x}}$ and $K_{x}=\left(\widehat{\mathcal{O}_{x}}\right)_{0}$, and the ring of adeles $\mathbb{A}_{C}$ is:

$$
\begin{aligned}
\mathbb{A}_{C} & =\prod_{x \in C}^{\prime} K_{x} \\
& =\left\{f=\left(f_{x}\right) \text { such that } f_{x} \in K_{x} \text { and } f_{x} \in A_{x} \text { for almost all } x\right\} .
\end{aligned}
$$

If $\mathbb{A}_{C}^{+}=\prod_{x \in C} A_{x}$, we can consider the $k$-vector spaces $\mathcal{V}=\mathbb{A}_{C}^{n}=$ $\prod_{x \in C}{ }^{\prime} K_{x}^{n}$ and $\mathcal{V}_{+}=\left(\mathbb{A}_{C}^{+}\right)^{n}=\prod_{x \in C} A_{x}^{n}$, and it is clear that $\operatorname{Gl}\left(n, \mathbb{A}_{C}\right) \subseteq$ $G_{\left(\mathbb{A}_{C}^{+}\right)^{n}}^{\mathbb{A}^{n}}$. It therefore follows from extension (3.1) that there exists a central extension of groups:

$$
1 \longrightarrow k^{*} \longrightarrow \widetilde{\operatorname{Gl}\left(n, \mathbb{A}_{C}\right)} \longrightarrow \operatorname{Gl}\left(n, \mathbb{A}_{C}\right) \longrightarrow 1 .
$$

Thus, the "Abstract Reciprocity Law" proved in $[\mathbf{6}]$ shows that the central extension of groups

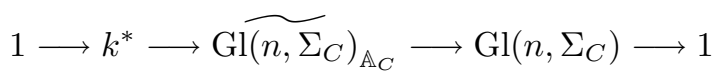

induced, through the diagonal immersion $\delta: \operatorname{Gl}\left(n, \Sigma_{C}\right) \hookrightarrow \operatorname{Gl}\left(n, \mathbb{A}_{C}\right)$, by the central extension (3.4) is trivial. 
Again according to the statements of [8], one has that:

Theorem 3.11 (Reciprocity Law). If $C$ is a complete, non-singular and irreducible curve over an algebraically closed field $k, G$ is an abelian group and $\psi: k^{*} \rightarrow G$ is a morphism of groups, given two commuting matrices $\tau, \sigma \in \operatorname{Gl}\left(n, \Sigma_{C}\right)$ one has that:

$$
\prod_{x \in C}(\tau, \sigma)_{x, \psi}^{n}=1
$$

where $(,)_{x, \psi}^{n}$ is the "tame symbol" on $\operatorname{Gl}\left(n, \Sigma_{C}\right)$ associated with the closed point $x$ and the morphism $\psi$.

\section{Parshin symbol on $\operatorname{Gl}\left(n, \Sigma_{S}\right)$}

Let $C$ be an irreducible and non-singular algebraic curve on a smooth, proper, geometrically irreducible surface $S$ over an algebraically closed field $k$. If $\Sigma_{S}$ is the function field of $S$, the curve $C$ defines a discrete valuation

$$
v_{C}: \Sigma_{S}^{*} \longrightarrow \mathbb{Z},
$$

whose residue class field is $\Sigma_{C}$ (the function field of $C$ ).

Thus, it follows from the central extension (3.2) that there exists a central extension of groups:

$$
1 \longrightarrow \Sigma_{C}^{*} \stackrel{i}{\longrightarrow} \mathrm{Gl}\left(\tilde{\left(n, \Sigma_{S}\right.}\right) \stackrel{\pi}{\longrightarrow} \mathrm{Gl}\left(n, \Sigma_{S}\right) \longrightarrow 1
$$

and its commutator will be denoted by $\{,\}_{v_{C}}^{n}$.

Now setting an element $z \in \Sigma_{S}^{*}$ with $v_{C}(z)=1$, we denote

$$
v_{x}^{n, z}(\tau)=i_{k}\left(\left\{\tau, \sigma_{z}^{n}\right\}_{v_{C}}^{n}, A_{x}\right)=v_{x}\left(\left\{\tau, \sigma_{z}^{n}\right\}_{v_{C}}^{n}\right) \in \mathbb{Z}
$$

for a matrix $\tau \in \operatorname{Gl}\left(n, \Sigma_{S}\right)$.

If we consider another element $z^{\prime} \in \Sigma_{S}^{*}$, with $v_{C}\left(z^{\prime}\right)=1$, there exists a transformation:

$$
v_{x}^{n, z^{\prime}} \longmapsto v_{x}^{n, z}+\lambda \cdot v_{C},
$$

with $\lambda=-v_{x}\left(\left\{z, z^{\prime}\right\}_{v_{C}}^{n}\right) \in \mathbb{Z}$.

Using the two interpretations of the Parshin symbol on $\Sigma_{S}$ as iterated tame symbols (Subsection 2.1), we shall now study two different generalizations of the Parshin symbol to the group $\operatorname{Gl}\left(n, \Sigma_{S}\right)$. 
4.1. First approach to a Parshin symbol on $\operatorname{Gl}\left(n, \Sigma_{S}\right)$. If $\{,\}_{A_{x}}^{K_{x}}$ is the commutator referred to in Example 2, we define the map:

$$
\{,\}_{x, C}^{n, z}: \operatorname{Gl}\left(n, \Sigma_{S}\right) \times \operatorname{Gl}\left(n, \Sigma_{S}\right) \times \operatorname{Gl}\left(n, \Sigma_{S}\right) \longrightarrow k^{*}
$$

by the expression:

$$
\begin{aligned}
\left\{\tau_{1}, \tau_{2}, \tau_{3}\right\}_{x, C}^{n, z}= & {\left[\left\{\left\{\tau_{1}, \sigma_{z}^{n}\right\}_{v_{C}}^{n},\left\{\tau_{2}, \sigma_{z}^{n}\right\}_{v_{C}}^{n}\right\}_{A_{x}}^{K_{x}}\right]^{-v_{C}\left(\operatorname{det} \tau_{3}\right)} } \\
= & (-1)^{(n-1) \cdot\left[v_{C}\left(\operatorname{det} \tau_{1}\right)+v_{C}\left(\operatorname{det} \tau_{2}\right)\right] v_{C}\left(\operatorname{det} \tau_{3}\right)} \\
& \cdot\left[\frac{\left(\operatorname{det} \tau_{1}\right)^{-v_{C}\left(\operatorname{det} \tau_{3}\right) \cdot v_{x}^{n, z}\left(\tau_{2}\right)}}{\left(\operatorname{det} \tau_{2}\right)^{-v_{C}\left(\operatorname{det} \tau_{3}\right) \cdot v_{x}^{n, z}\left(\tau_{1}\right)}}(p)\right]
\end{aligned}
$$

for all matrices $\tau_{1}, \tau_{2}, \tau_{3} \in \operatorname{Gl}\left(n, \Sigma_{S}\right)$.

Thus, analogously to (2.4), we can consider the map:

$$
\begin{aligned}
& \left\{\tau_{1}, \tau_{2}, \tau_{3}\right\}_{x, C}^{n}=\left\{\tau_{1}, \tau_{2}, \tau_{3}\right\}_{x, C}^{n, z} \cdot\left\{\tau_{2}, \tau_{3}, \tau_{1}\right\}_{x, C}^{n, z} \cdot\left\{\tau_{3}, \tau_{1}, \tau_{2}\right\}_{x, C}^{n, z} \\
& =\left[\left(\operatorname{det} \tau_{1}\right)^{v_{C}\left(\operatorname{det} \tau_{2}\right) \cdot v_{x}^{n, z}\left(\tau_{3}\right)-v_{C}\left(\operatorname{det} \tau_{3}\right) \cdot v_{x}^{n, z}\left(\tau_{2}\right)}\right.
\end{aligned}
$$

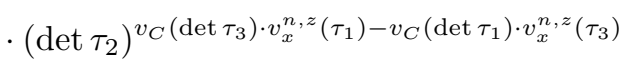

$$
\begin{aligned}
& \left.\cdot\left(\operatorname{det} \tau_{3}\right)^{v_{C}\left(\operatorname{det} \tau_{1}\right) \cdot v_{x}^{n, z}\left(\tau_{2}\right)-v_{C}\left(\operatorname{det} \tau_{2}\right) \cdot v_{x}^{n, z}\left(\tau_{1}\right)}\right](p) \\
& =\left\{\operatorname{det} \tau_{1}, \operatorname{det} \tau_{2}, \operatorname{det} \tau_{3}\right\}_{x, C},
\end{aligned}
$$

for all matrices $\tau_{1}, \tau_{2}, \tau_{3} \in \operatorname{Gl}\left(n, \Sigma_{S}\right),\{,,\}_{x, C}$ being the map referred to in Subsection 2.1. Hence, $\{\cdot, \cdot, \cdot\}_{x, C}^{n}$ is independent of the choice of the parameter $z$.

If we consider the symbol:

$$
\langle,,\rangle_{x, C}^{n}: \operatorname{Gl}\left(n, \Sigma_{S}\right) \times \operatorname{Gl}\left(n, \Sigma_{S}\right) \times \mathrm{Gl}\left(n, \Sigma_{S}\right) \longrightarrow k^{*},
$$

defined as:

$$
\left\langle\tau_{1}, \tau_{2}, \tau_{3}\right\rangle_{x, C}^{n}=(-1)^{\alpha\left(\tau_{1}, \tau_{2}, \tau_{3}\right)} \cdot\left\{\tau_{1}, \tau_{2}, \tau_{3}\right\}_{x, C}^{n}
$$


with

$$
\begin{aligned}
\alpha\left(\tau_{1}, \tau_{2}, \tau_{3}\right)= & v_{C}\left(\operatorname{det} \tau_{1}\right) \cdot v_{C}\left(\operatorname{det} \tau_{2}\right) \cdot v_{x}^{n, z}\left(\tau_{3}\right) \\
& +v_{C}\left(\operatorname{det} \tau_{1}\right) \cdot v_{C}\left(\operatorname{det} \tau_{3}\right) \cdot v_{x}^{n, z}\left(\tau_{2}\right) \\
& +v_{C}\left(\operatorname{det} \tau_{2}\right) \cdot v_{C}\left(\operatorname{det} \tau_{3}\right) \cdot v_{x}^{n, z}\left(\tau_{1}\right) \\
& +v_{C}\left(\operatorname{det} \tau_{1}\right) \cdot v_{x}^{n, z}\left(\tau_{2}\right) \cdot v_{x}^{n, z}\left(\tau_{3}\right) \\
& +v_{C}\left(\operatorname{det} \tau_{2}\right) \cdot v_{x}^{n, z}\left(\tau_{1}\right) \cdot v_{x}^{n, z}\left(\tau_{3}\right) \\
& +v_{C}\left(\operatorname{det} \tau_{3}\right) \cdot v_{x}^{n, z}\left(\tau_{1}\right) \cdot v_{x}^{n, z}\left(\tau_{2}\right)
\end{aligned}
$$

we have that this symbol generalizes the Parshin symbol on $\Sigma_{S}$. However, since the integer number $v_{x}^{n, z}(\tau)$ coincides with the valuation $v_{x}^{z}(\operatorname{det} \tau)$ referred to in Subsection 2.1, there exists a commutative diagram

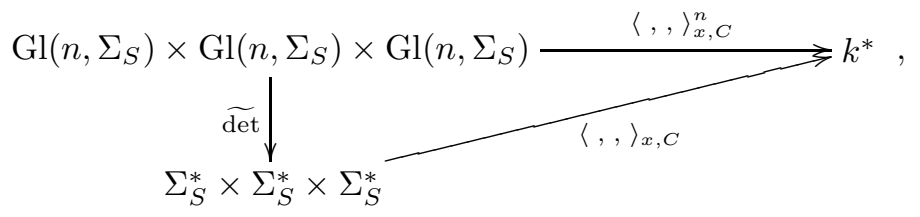

where $\widetilde{\operatorname{det}}\left(\tau_{1}, \tau_{2}, \tau_{3}\right)=\left(\operatorname{det} \tau_{1}, \operatorname{det} \tau_{2}, \operatorname{det} \tau_{3}\right)$ and $\langle,,\rangle_{x, C}$ is the Parshin symbol associated with the sequence $x \in C \subset S$. Hence, the symbol $\langle,,\rangle_{x, C}^{n}$ is not a new symbol, and it follows from the properties of the Parshin symbol on an algebraic surface that

$$
\begin{aligned}
& \prod_{x}\left\langle\tau_{1}, \tau_{2}, \tau_{3}\right\rangle_{x, C}^{n}=1, \\
& \prod_{C}\left\langle\tau_{1}, \tau_{2}, \tau_{3}\right\rangle_{x, C}^{n}=1,
\end{aligned}
$$

where $C$ is a complete, irreducible and non-singular curve in (4.2), and the second reciprocity law is the product over all irreducible curves containing a fixed point $x \in S$.

4.2. Second approach to a Parshin symbol on $\operatorname{Gl}\left(n, \Sigma_{S}\right)$. If $z$ is again a function $z$ on $S$ such that $v_{C}(z)=1$, and $t$ is a function on $C$ such that $v_{x}(t)=1$, given three commuting matrices $\tau_{1}, \tau_{2}, \tau_{3} \in \operatorname{Gl}\left(n, \Sigma_{S}\right)$ we can define the assignment:

$$
\left\{\tau_{1}, \tau_{2}, \tau_{3}\right\}_{x, C}^{n, z, t}=\left[\left\{\left\{\tau_{1}, \tau_{2}\right\}_{v_{C}}^{n}, t\right\}_{A_{x}}^{K_{x}}\right]^{v_{x}^{n, z}\left(\tau_{3}\right)} \in k^{*},
$$


and, similar to $(2.5)$, we write:

$$
\left\{\left\{\tau_{1}, \tau_{2}, \tau_{3}\right\}\right\}_{x, C}^{n, z, t}=\left\{\tau_{1}, \tau_{2}, \tau_{3}\right\}_{x, C}^{n, z, t} \cdot\left\{\tau_{2}, \tau_{3}, \tau_{1}\right\}_{x, C}^{n, z, t} \cdot\left\{\tau_{3}, \tau_{1}, \tau_{2}\right\}_{x, C}^{n, z, t} .
$$

Bearing in mind that the commutator of a central extension is a bimultiplicative map, one has that $\{\{,,\}\}_{x, C}^{n, z, t}$ is multiplicative in each argument:

$$
\begin{aligned}
&\left\{\left\{\tau_{1} \cdot \tau_{2}, \sigma, \varphi\right\}\right\}_{x, C}^{n, z, t}=\left\{\left\{\tau_{1}, \sigma, \varphi\right\}\right\}_{x, C}^{n, z, t} \cdot\left\{\left\{\tau_{2}, \sigma, \varphi\right\}\right\}_{x, C}^{n, z, t} \\
&\left\{\left\{\tau, \sigma_{1} \cdot \sigma_{2}, \varphi\right\}\right\}_{x, C}^{n, z, t}=\left\{\left\{\tau, \sigma_{1}, \varphi\right\}\right\}_{x, C}^{n, z, t} \cdot\left\{\left\{\tau, \sigma_{2}, \varphi\right\}\right\}_{x, C}^{n, z, t} \\
&\left\{\left\{\tau, \sigma, \varphi_{1} \cdot \varphi_{2}\right\}\right\}_{x, C}^{n, z, t}=\left\{\left\{\tau, \sigma, \varphi_{1}\right\}\right\}_{x, C}^{n, z, t} \cdot\left\{\left\{\tau, \sigma, \varphi_{2}\right\}\right\}_{x, C}^{n, z, t}
\end{aligned}
$$

for commuting matrices $\tau_{i}, \sigma_{i}, \varphi_{i} \in \mathrm{Gl}\left(n, \Sigma_{S}\right)$.

Moreover, since the commutator of a central extension is skew-symmetric, the map $\{\{,,\}\}_{x, C}^{n, z, t}$ satisfies the property:

$$
\{\{\tau, \tau, \sigma\}\}_{x, C}^{n, z, t}=\{\{\tau, \sigma, \tau\}\}_{x, C}^{n, z, t}=\{\{\sigma, \tau, \tau\}\}_{x, C}^{n, z, t}=1
$$

for all commuting matrices $\tau, \sigma \in \operatorname{Gl}\left(n, \Sigma_{S}\right)$.

Furthermore, from the definition of $\{\{,,\}\}_{x, C}^{n, z, t}$, one also has that:

$$
\left\{\left\{\tau_{\widetilde{\sigma}(1)}, \tau_{\widetilde{\sigma}(2)}, \tau_{\widetilde{\sigma}(3)}\right\}\right\}_{x, C}^{n, z, t}=\left[\left\{\left\{\tau_{1}, \tau_{2}, \tau_{3}\right\}\right\}_{x, C}^{n, z, t}\right]^{\operatorname{sign} \widetilde{\sigma}}
$$

for any permutation $\widetilde{\sigma}$, and commuting matrices $\tau_{i} \in \operatorname{Gl}\left(n, \Sigma_{S}\right)$.

Remark 4.1. If $f, g, h \in \Sigma_{S}^{*}$, one has that

$$
\left\{\left\{\sigma_{f}^{n}, \sigma_{g}^{n}, \sigma_{h}^{n}\right\}\right\}_{x, C}^{n, z, t}=\left[\{f, g, h\}_{x, C}\right]^{n^{2}} .
$$

The map $\{\{\cdot, \cdot, \cdot\}\}_{x, C}^{n, z, t}$ does not therefore factorize through the determinant map det.

However,

Proposition 4.2. The map $\{\{\cdot, \cdot, \cdot\}\}_{x, C}^{n, z, t}$ depends on the parameter $t$.

Proof: If we consider three diagonal matrices $\tau_{f_{i}}, \tau_{g_{j}}, \tau_{h_{k}} \in \operatorname{Gl}\left(n, \Sigma_{S}\right)$, where

$$
\tau_{f_{i}}=\left(\begin{array}{ccc}
f_{1} & \ldots & 0 \\
\vdots & \ddots & \vdots \\
0 & \ldots & f_{n}
\end{array}\right), \tau_{g_{j}}=\left(\begin{array}{ccc}
g_{1} & \ldots & 0 \\
\vdots & \ddots & \vdots \\
0 & \ldots & g_{n}
\end{array}\right) \text { and } \tau_{h_{k}}=\left(\begin{array}{ccc}
h_{1} & \ldots & 0 \\
\vdots & \ddots & \vdots \\
0 & \ldots & h_{n}
\end{array}\right) \text {, }
$$


it follows from Example 1 that the explicit expression of the map is:

$$
\begin{aligned}
& \left\{\left\{\tau_{f_{i}}, \tau_{g_{j}}, \tau_{h_{k}}\right\}\right\}_{x, C}^{n, z, t}=\prod_{s=1}^{n}\left[f_{s}^{v_{C}\left(g_{s}\right) \cdot v_{x}^{n, z}\left(\tau_{h_{k}}\right)-v_{C}\left(h_{s}\right) \cdot v_{x}^{n, z}\left(\tau_{g_{j}}\right)}\right.
\end{aligned}
$$

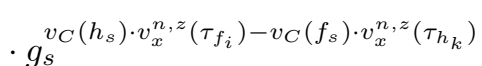

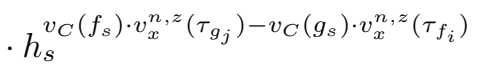

$$
\begin{aligned}
& \left.\cdot t^{\beta_{s}\left(z, \tau_{f_{i}}, \tau_{g_{j}}, \tau_{h_{k}}\right)}\right](x),
\end{aligned}
$$

with

$$
\begin{aligned}
\beta_{s}\left(z, \tau_{f_{i}}, \tau_{g_{j}}, \tau_{h_{k}}\right)= & {\left[v_{x}^{z}\left(f_{s}\right) v_{c}\left(g_{s}\right)-v_{x}^{z}\left(g_{s}\right) v_{c}\left(f_{s}\right)\right] v_{x}^{n, z}\left(\tau_{h_{k}}\right) } \\
& +\left[v_{x}^{z}\left(g_{s}\right) v_{c}\left(h_{s}\right)-v_{x}^{z}\left(h_{s}\right) v_{c}\left(g_{s}\right)\right] v_{x}^{n, z}\left(\tau_{f_{i}}\right) \\
& +\left[v_{x}^{z}\left(h_{s}\right) v_{c}\left(f_{s}\right)-v_{x}^{z}\left(f_{s}\right) v_{c}\left(h_{s}\right)\right] v_{x}^{n, z}\left(\tau_{g_{j}}\right),
\end{aligned}
$$

and $v_{x}^{n, z}\left(\tau_{\varphi_{d}}\right)=\sum_{d=1}^{n} v_{x}^{z}\left(\varphi_{d}\right)$

Bearing in mind that

$$
\begin{aligned}
\sum_{s=1}^{n} \beta_{s}\left(z, \tau_{f_{i}}, \tau_{g_{j}}, \tau_{h_{k}}\right)= & \sum_{s \neq r}\left[\left[v_{x}^{z}\left(f_{s}\right) v_{c}\left(g_{s}\right)-v_{x}^{z}\left(g_{s}\right) v_{c}\left(f_{s}\right)\right] v_{x}^{z}\left(h_{r}\right)\right. \\
& +\left[v_{x}^{z}\left(g_{s}\right) v_{c}\left(h_{s}\right)-v_{x}^{z}\left(h_{s}\right) v_{c}\left(g_{s}\right)\right] v_{x}^{z}\left(f_{r}\right) \\
& \left.+\left[v_{x}^{z}\left(h_{s}\right) v_{c}\left(f_{s}\right)-v_{x}^{z}\left(f_{s}\right) v_{c}\left(h_{s}\right)\right] v_{x}^{z}\left(g_{r}\right)\right] \neq 0
\end{aligned}
$$

the statement is proved.

Corollary 4.3. The map $\{\{\cdot, \cdot, \cdot\}\}_{x, C}^{n, z, t}$ does not allow us to define a generalization of the Parshin symbol to the linear group $\operatorname{Gl}\left(n, \Sigma_{S}\right)$.

Remark 4.4. A remaining problem is to characterize the explicit expression of the commutator $\{\tau, \sigma\}_{\mathcal{V}_{+}^{n}}^{\mathcal{V}^{n}}$ for all commuting matrices $\tau, \sigma \in$ $\operatorname{Gl}(n, \mathcal{F})$. This explicit expression will be a basic tool for determining a possible 2-dimensional symbol on $\operatorname{Gl}\left(n, \Sigma_{S}\right)$ generalizing the Parshin symbol on an algebraic surface.

\section{References}

[1] E. Arbarello, C. De Concini and V. G. Kac, The infinite wedge representation and the reciprocity law for algebraic curves, in: "Theta functions-Bowdoin 1987", Part 1 (Brunswick, ME, 1987), Proc. Sympos. Pure Math. 49, Amer. Math. Soc., Providence, RI, 1989, pp. 171-190. 
[2] A. Beilinson, S. Bloch And H. Esnault, $\epsilon$-factors for GaussManin determinants, Dedicated to Yuri I. Manin on the occasion of his 65th birthday, Mosc. Math. J. 2(3) (2002), 477-532.

[3] J.-L. Brylinski And D. A. MCLaughlin, The geometry of twodimensional symbols, $K$-Theory 10(3) (1996), 215-237.

[4] C. Contou-Carrère, Jacobienne locale, groupe de bivecteurs de Witt universel, et symbole modéré, C. R. Acad. Sci. Paris Sér. I Math. 318(8) (1994), 743-746.

[5] I. B. Fesenko, A generalized symbol in multidimensional local fields, (Russian), in: "Rings and modules. Limit theorems of probability theory, No. 2", (Russian), Leningrad. Univ., Leningrad, 1988, pp. 88-92, 214.

[6] J. M. Muñoz Porras and F. Pablos Romo, Generalized reciprocity laws, Trans. Amer. Math. Soc. (to appear).

[7] F. Pablos Romo, Algebraic construction of the tame symbol and the Parshin symbol on a surface, J. Algebra 274(1) (2004), 335-346.

[8] F. Pablos Romo, Central extensions, symbols and reciprocity laws on $\operatorname{Gl}(n, \mathcal{F})$, Preprint, Universidad de Salamanca (2005).

[9] F. Pablos Romo, On the tame symbol of an algebraic curve, Comm. Algebra 30(9) (2002), 4349-4368.

[10] A. N. Parshin, Local class field theory, (Russian), Algebraic geometry and its applications, Trudy Mat. Inst. Steklov. 165 (1984), $143-170$.

[11] J. TATE, Residues of differentials on curves, Ann. Sci. École Norm. Sup. (4) 1 (1968), 149-159.

[12] S. V. Vostokov and I. B. Fesenko, Torsion in Milnor's higher functors for multidimensional local fields, (Russian), in: "Rings and modules. Limit theorems of probability theory, No. 1", (Russian), Leningrad. Univ., Leningrad, 1986, pp. 75-87, 213.

Departamento de Matemáticas

Universidad de Salamanca

Plaza de la Merced 1-4

37008 Salamanca

Spain

E-mail address: fpablos@usal.es 